\title{
Modeling Loss of Microvascular Wall Homeostasis during Glycocalyx Deterioration and Hypertension that Impacts Plasma Filtration and Solute Exchange
}

\author{
Laura Facchini $^{* \mathrm{a}}$, Alberto Bellin ${ }^{\mathrm{b}}$ and Eleuterio F. Toro ${ }^{\mathrm{c}}$ \\ ${ }^{a}$ Department of Mathematics, University of Trento, Italy; ${ }^{b}$ Department of Civil, Environmental and Mechanical \\ Engineering, University of Trento, Italy; ${ }^{c}$ Laboratory of Applied Mathematics, DICAM, University of Trento, Italy
}

\begin{abstract}
The fiber matrix of the surface glycocalyx layer internally coating the endothelial cells and plugging the intercellular clefts is crucial for microvascular wall homeostasis. Disruption of the glycocalyx is found in clinical conditions characterized by microvascular and endothelial dysfunction such as atherosclerosis, diabetes mellitus, chronic renal failure and cerebrovascular disease. Shedding of its components may also occur during oxidative stress and systemic inflammatory states including septis. In this work, we investigate the effects of glycocalyx degradation, either due to enzymatic digestion or to agonist recruitment, on plasma filtration and solute extravasation. We also take into account the possibility of a physiological or pathological increase in blood pressure, as in hypertensive zones such as pre- and post-stenotic blood vessels. Our mathematical model shows that a seriously damaged glycocalyx produces an augmentation of flux of both solvent and solute, thus losing its role of transport barrier and macro-molecular sieve, in agreement with experimental evidence. Similarly, hypertension causes an increase in both volume and solute fluxes, also according to physiological findings. The combination of glycocalyx deterioration and hypertension further raises plasma and solute fluxes, potentially leading in most severe cases to edema and hemorrhage, as in the case of diabetes.
\end{abstract}

Keywords: Filtration; Solute transport; Time-dependent; One-dimensional; Multi-layer; Hemorrhage.

\section{INTRODUCTION}

A pathological loss or agonist-induced thinning of glycocalyx, a single-cell-thick layer currently recognized as crucial for microvascular wall homeostasis [1-6], may impair vascular wall protection throughout the circulatory system [5]. In particular, experimental studies in which the glycocalyx was treated with glycosaminoglycan-degrading enzymes showed that enzymatic degradation results in (a) reduction of anionic dextran exclusion [7], (b) formation of perivascular edema [8], (c) impairment of shear stress-dependent NO production in arteries and cultured endothelial cells [9, 10], (d) increase in hydraulic conductivity [11], protein permeability [12], albumin glomerular clearance [13] and adhesion of platelets and leukocytes in venules [14].

Degradation or loss of the glycocalyx has been observed during exposure to atherogenic/cardiovascular risk factors, including inflammatory and atherogenic stimuli, ischemia/reperfusion and oxidized low-density lipoprotein infusion [5]. On the other hand, glycocalyx proteins are damaged in vascular diseases, leading to inflammation, which in turn causes further damages in a sort of vicious circle [15]. Patients with type 1 diabetes mellitus and microalbuminuria have been shown to be more susceptible to glycocalyx degradation, compared to their normal buminuric counterparts [16]. Glycocalyx disruption occurs also in clinical conditions, such as chronic renal failure, cerebrovascular disease and septis [17-21] and in high-fat high-cholesterol diet [22]. Moreover, this loss of systemic glycocalyx volume, leading to fluid leakage from microvessels, correlates to a reduction in anatomic capillary diameter or functional capillary density [5, 23]. Glycocalyx degeneration, induced by reactive oxygen species exposure, has been shown to lead to endothelial dysfunction, with possible onset of microalbuminuria or proteinuria (i.e. the presence of an excess of albumin or other serum proteins in the urine) in clinical diseases [24, 25], and subsequently to vascular inflammation and cardiovascular disease progression [26].

Moreover, shear stress appears to contribute both to the incorporation of the glycosaminoglycan hyaluronan into the glycocalyx, thus affecting sulfated glycosaminoglycan distribution, and to the shedding of hyaluronan (for instance, during reperfusion after ischemia), thus permitting leukocyte adherence to the vessel wall [5]. Shear stress seems to be mediated by NO production [10] and G proteins [27]. Another factor, which is crucial for microvascular wall homeostasis, is capillary pressure. In particular, hypertension plays a role in trans-vascular exchange, increasing both fluid filtration and protein extravasation. This leads in most severe cases to leakage, as in the cases of extra-vascular edema and hemorrhage, which are observed for instance in hypertensive retinopathy and hypertensive optic neuropathy [28]. Systolic blood pressure higher than $115 \mathrm{mmHg}$ is responsible for $62 \%$ of cerebrovascular disease (including stroke, myocardial infarction, heart failure, aneurysms of the arteries, coronary heart disease and peripheral arterial disease) and $49 \%$ of ischemic heart disease.

A way to study the effect of both glycocalyx shedding and pressure increase on transvascular exchange is through the construction of a mathematical model, able to describe the effect of increasing glycocalyx permeability or internal pressure on plasma and solute fluxes. 
Starting from the 50s one- [29-31] and three-dimensional [30-34] mathematical models have been developed, by using different strategies to include the effects of glycocalyx and endothelial cells [1, 35]. Squire et al. [3] proposed a quasiperiodic three-dimensional ultra-structural model of the endothelial surface layer, which was subsequently used in [6] to study the flexural rigidity of the core proteins comprising the bush-like matrix and the adhesive molecular interactions between proteins in the endothelial membrane and circulating cellular components. In order to incorporate charge effect on microvessel permeability, the model developed in [34] was extended in [36] to include a negatively charged glycocalyx layer at the entrance of the interendothelial cleft. In the present paper only the size-filtering capacity of the glycocalyx is considered, assuming that the charge-sieving effect can be neglected. The effects of glycocalyx thinning and of a change in its morphology are also described in [37], in which the glycocalyx enters into the mathematical model simply via the boundary conditions.

In the present paper we study, by means of our mathematical model [38], the effect of glycocalyx deterioration and blood pressure alterations, on filtration and transport of macromolecules. Our model differs from existing studies in the way the main structural elements are described, thereby allowing to explicitly compute the distribution of hydrostatic and osmotic pressure inside the vessel wall, without lumping glycocalyx and the layer of endothelial cells into a single layer, as done in most of the existing models. This allows a more accurate reconstruction of plasma filtration and solute leakage, also in the interface region between the glycocalyx and the endothelium. For the purpose of the present paper we consider a typical extra-cranial capillary, composed by two homogeneous, yet different, layers representing the glycocalyx and the endothelium.

The rest of the paper is structured as follows. In Section 2, the steady-state mathematical model of plasma filtration and solute transport across a typical microvessel wall is summarized, while Section 3 recalls the physiological parameters. The main results of this paper are reported in Sections 4 and 5, while Section 6 draws some conclusions.

\section{THE MATHEMATICAL MODEL}

Assuming radial symmetry, we model steady-state plasma filtration and solute transport across the vessel wall by means of the following coupled non-linear ordinary differential equations [38], written in cylindrical coordinates $(\mathrm{r}, \theta, \mathrm{z})$ and in dimensionless form:

$$
\left\{\begin{array}{c}
\frac{\mathrm{d}}{\mathrm{d} r}\left[(r+\xi) \ell_{p}\left(\frac{\mathrm{d} p}{\mathrm{~d} r}-\sigma \frac{\mathrm{d} \Pi}{\mathrm{d} r}\right)\right]=0 \\
\frac{\mathrm{d}}{\mathrm{d} r}\left[(r+\xi) \Pi\left(A \frac{\mathrm{d} p}{\mathrm{~d} r}+B \frac{\mathrm{d} \Pi}{\mathrm{d} r}\right)\right]=0
\end{array}\right.
$$

Where both the hydrostatic pressure $p=p(r)$ and the osmotic pressure $\Pi=\Pi(\mathrm{r})$ are functions of the dimensionless distance $r$ from the vessel axis, with $r \in(0,1)$. From a mathematical viewpoint, Equations (1) are a two-point boundary-value problem with boundary conditions:

$$
\mathrm{p}_{\mathrm{c}}=\mathrm{p}(0) ; \quad \mathrm{p}_{\mathrm{o}}=\mathrm{p}(1) ; \quad \Pi_{\mathrm{c}}=\Pi(0) ; \quad \Pi_{\mathrm{o}}=\Pi(1) .
$$

The dimensionless quantities are defined as follows:

$$
\begin{aligned}
& p=\frac{p^{*}}{p_{R}}, \quad \Pi=\frac{\Pi^{*}}{p_{R}}, \quad \xi=\frac{r_{c}}{r_{o}-r_{c}}, \quad r=\frac{r^{*}-r_{c}}{r_{o}-r_{c}}, \\
& \ell_{p}=\frac{\ell_{p}^{*}}{\ell_{p}^{H}}, \quad \ell_{d}=\frac{\ell_{d}^{*}}{\ell_{p}^{H}}, \quad A=\frac{\ell_{p}^{*}(\sigma-1)}{\ell_{p}^{H}}, \quad B=\frac{\ell_{p}^{*} \sigma-\ell_{d}^{*}}{\ell_{p}^{H}},
\end{aligned}
$$

where the superscript $*$ denotes dimensional quantities, $\mathrm{r}_{\mathrm{o}}$ and $r_{c}$ are the internal and the external radius (dimensional) of the vessel wall, respectively, the former corresponding to the internal surface of the glycocalyx. Furthermore, $\ell_{\mathrm{p}}^{\mathrm{H}}$ is the harmonic mean of the hydraulic conductivities of the glycocalyx $\left(\ell_{\mathrm{p}}^{\mathrm{G}}\right)$ and the endothelial cells $\left(\ell_{\mathrm{p}}^{\mathrm{w}}\right)$, weighted by their thickness:

$$
\ell_{p}^{H}=\frac{r_{o}-r_{c}}{\frac{r_{g}-r_{c}}{\ell_{p}^{G}}+\frac{r_{o}-r_{g}}{\ell_{p}^{W}}},
$$

where $r_{g}$ is the radius of the circle representing the external glycocalyx surface. $\sigma$ is the membrane reflection coefficient and $\ell_{\mathrm{d}}$ is the diffusional permeability. We choose as reference pressure $p_{R}$ the magnitude of the external hydrostatic pressure $\left|p_{o}{ }^{*}\right|$.

We solve system (1) for $\mathrm{p}(\mathrm{r})$ and $\Pi(\mathrm{r})$ using the finite difference scheme proposed in [39]. Then we compute the dimensionless volume $J_{v}=J_{v}(r)$ and solute $J_{s}=J_{s}(r)$ fluxes by using the following expressions:

$$
\begin{aligned}
& J_{v}=-2 \pi(r+\xi) \ell_{p}\left(\frac{\mathrm{d} p}{\mathrm{~d} r}-\sigma \frac{\mathrm{d} \Pi}{\mathrm{d} r}\right), \\
& J_{s}=2 \pi(r+\xi) \Pi\left[\ell_{p}(\sigma-1) \frac{\mathrm{d} p}{\mathrm{~d} r}+\left(\ell_{p} \sigma-\ell_{d}\right) \frac{\mathrm{d} \Pi}{\mathrm{d} r}\right] .
\end{aligned}
$$

At this stage, we note that even if system (1) implies that both fluxes are constant in $r$, because of numerical approximation, the solution shows small variations. We thus consider as flux, the mean of the numerical values across the vessel thickness.

\section{PHYSIOLOGICAL PARAMETERS}

\subsection{Relation between parameters}

We model the glycocalyx as a system of $\mathrm{N}$ cylindrical pores (per unit area of surface membrane) of mean radius $r_{p}$. According to this conceptual model, the phenomenological parameters of the membrane representing the glycocalyx assume the following expressions [40]:

$$
\begin{aligned}
\sigma & =(1-\varphi)^{2}, \quad \text { with } \varphi=\left(1-\frac{r_{s}}{r_{p}}\right)^{2}, \\
\ell_{p} & =\frac{N \pi r_{p}^{4}}{8 \mu}, \\
\ell_{d} & =\frac{N \pi r_{p}^{2} D \varphi}{\Pi_{M}} f_{p}\left(\frac{r_{s}}{r_{p}}\right),
\end{aligned}
$$

where $\sigma \in[0,1]$ is the reflection coefficient, $\varphi$ is the solute partition coefficient (which represents the reduction of diffusion due to steric exclusion), $\mathrm{r}_{\mathrm{s}}$ is the radius of the solute 
molecule, $\ell_{\mathrm{p}}$ is the hydraulic conductivity, $\mu$ is the kinematic fluid (plasma) viscosity, $\ell_{\mathrm{d}}$ is the diffusional permeability, $\mathrm{D}$ is the diffusion coefficient of the solute in aqueous solution and $\Pi_{\mathrm{M}}$ is a mean osmotic pressure (here assumed as the arithmetic mean of the values assigned as boundary conditions); $\mathrm{f}_{\mathrm{p}}$ is an empirical function introduced to take into account the restricted diffusion due to the increased hydrodynamic drag [41]:

$$
f_{p}(x)=1-2.104 x+2.09 x^{3}-0.95 x^{5} .
$$

Note that all the previous quantities are dimensional.

Considerations from irreversible thermodynamics [42] lead to:

$$
\ell_{\mathrm{d}}>\sigma^{2} \ell_{\mathrm{p}}
$$

We thus assume that the diffusional permeability is given by $\ell_{\mathrm{d}}=\alpha \sigma^{2} \ell_{\mathrm{p}}$, where $\alpha>1$ is a constant related to the Péclet number Pe $=\ell_{\mathrm{p}} / \ell_{\mathrm{d}}$ through $\alpha=1 /\left(\sigma^{2} \mathrm{Pe}\right)$.

Manipulating the previous algebraic equations, the diffusional permeability can be expressed as a function of the reflection coefficient and the hydraulic conductivity as

$$
\ell_{d}=\frac{8 \mu D}{r_{p}^{2} \Pi_{M}} \ell_{p}(1-\sqrt{\sigma}) f_{p}(1-\sqrt{1-\sqrt{\sigma}})
$$

where the radius $r_{p}$ and the number of the pores can be estimated through the following expressions

$$
\begin{aligned}
r_{p} & =\frac{r_{s}}{1-\sqrt{1-\sqrt{\sigma_{G}}}}, \\
N & =\frac{8 \mu \ell_{p}^{G}}{\pi r_{p}^{4}},
\end{aligned}
$$

Suitable vales of $\sigma_{\mathrm{G}}$ and $\ell_{\mathrm{p}}{ }^{\mathrm{G}}$ of the glycocalyx layer are reported, for instance, in [43]. Note that the obtained mean pore radius $r_{p}=4.65 \mathrm{~nm}$ is comparable with the $4-5 \mathrm{~nm}$ halfdistance of the periodic glycocalyx surface structure determined from electron micrographs [3] and the $5.7 \mathrm{~nm}$ pore exclusion size limiting glycocalyx permeation by dextrans [44].

The effective diffusion coefficient $\mathrm{D}$ can be estimated from the free diffusion coefficient $D_{\text {free }}$ as $D=\theta_{p} D_{\text {free }}$, where the coefficient $\theta_{\mathrm{p}}>1$, defined as

$$
\theta_{p}=\frac{\alpha \sigma^{2} \Pi_{M} r_{p}^{2}}{8 \mu D_{\text {free }}(1-\sqrt{\sigma}) f_{p}(1-\sqrt{1-\sqrt{\sigma}})},
$$

represents the increase of diffusivity due the non-uniform distribution of the velocity profile within the pore channel. Often, to take into account the hydrodynamic origin of this increase, the modified diffusion coefficient is called hydrodynamic dispersion [45]. In our case, $\theta_{\mathrm{p}}$ is about 33, while when the size of the molecules are much more smaller that the diameter the effective diffusivity may become 3-4 times larger than the diffusion coefficient.
By defining $\theta_{\mathrm{p}}$ as in equation (14), we automatically satisfy condition (10).

Since the function $f_{p}$ is positive (within a reasonable domain) and $\ell_{\mathrm{d}}>0$, an increase in the hydraulic conductivity $\ell_{\mathrm{p}}$ produces a linear increase in $\ell_{\mathrm{d}}$, as depicted in Figure 1 . Equation (11) also states that a reduction of the reflection coefficient $\sigma$ produces an increase in the diffusional permeability $\ell_{\mathrm{d}}$ (for a reasonable range of $\sigma$, see Figure 1), as we expect from the augmented mobility of the solute molecules.

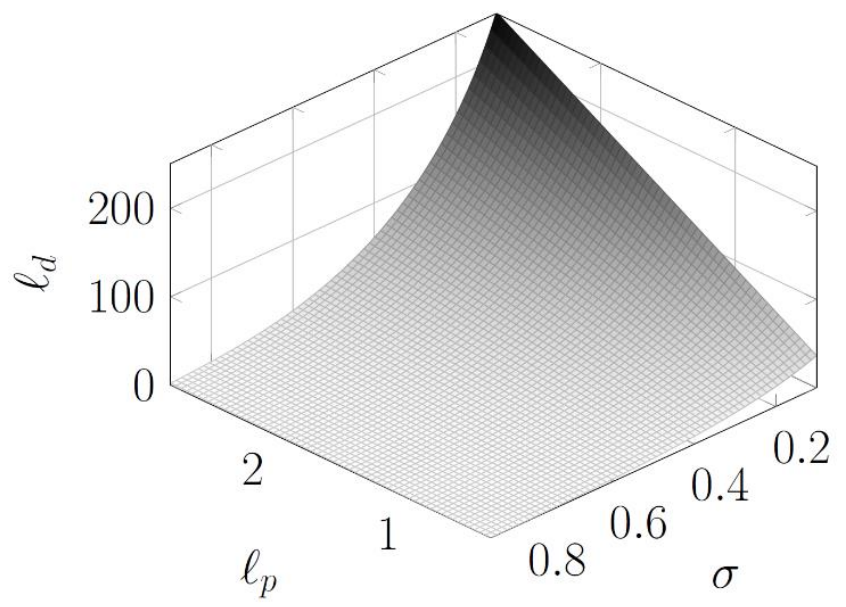

Figure 1. Diffusional permeability $\ell_{\mathrm{d}}$ versus $\ell_{\mathrm{p}}$ and $\sigma$. Here the physiological parameters $\ell_{\mathrm{p}}$ and $\ell_{\mathrm{d}}$ are dimensionless with respect to the weighted harmonic mean for the hydraulic conductivity $\ell_{\mathrm{p}}{ }^{\mathrm{H}}$.

\subsection{Physiological parameters across the wall}

In order to obtain smooth solutions, we consider a smooth transition between the two layers composing the vessel wall as in [38]:

$\left\{\begin{array}{l}\sigma(r)=\frac{\sigma_{G}+\sigma_{W}}{2}-\frac{\sigma_{G}-\sigma_{W}}{2} \frac{r-r_{g}}{\sqrt{\varepsilon^{2}+\left(r-r_{g}\right)^{2}}}, \\ \ell_{p}(r)=\frac{\ell_{p}^{G}+\ell_{p}^{W}}{2}-\frac{\ell_{p}^{G}-\ell_{p}^{W}}{2} \frac{r-r_{g}}{\sqrt{\varepsilon^{2}+\left(r-r_{g}\right)^{2}}},\end{array}\right.$

where sub-scripts $\mathrm{G}$ and $\mathrm{W}$ indicate the reflection coefficient of glycocalyx and the endothelial cells, respectively, and $r_{g}$ identifies the interface between the glycocalyx layer and the endothelium. We control the transition between the material properties of the two layers through the smoothing parameter $\varepsilon$. According to Equation (15), a larger $\varepsilon$ value results in a smoother transition. Here we choose $\varepsilon^{2}=10^{-5}$, thus the transition is assumed smooth, though sharp.

Table 1 shows typical values of material properties and physiological features of an intact capillary, as well as the values of the osmotic and hydrostatic pressures within the lumen and in the external interstitial space. The table reports also the physical and hydrodynamics properties of the solute (i.e. albumin), as well as kinematic fluid viscosity within the pores. 


\begin{tabular}{l|c|c} 
Parameter [unit] & Value & Reference \\
\hline $\mathrm{r}_{\mathrm{c}}[\mu \mathrm{m}]$ & 5 & {$[46]$} \\
$\mathrm{r}_{\mathrm{g}}[\mu \mathrm{m}]$ & 5.15 & {$[47]$} \\
$\mathrm{r}_{\mathrm{o}}[\mu \mathrm{m}]$ & 5.5 & {$[46]$} \\
$\Pi_{\mathrm{c}}[\mathrm{mmHg}]$ & 25 & {$[48]$} \\
$\Pi_{\mathrm{o}}[\mathrm{mmHg}]$ & 12 & {$[48]$} \\
$\mathrm{p}_{\mathrm{c}}[\mathrm{mmHg}]$ & 20 & {$[48]$} \\
$\mathrm{p}_{\mathrm{o}}[\mathrm{mmHg}]$ & -1 & {$[48]$} \\
$\sigma_{\mathrm{G}}$ & 0.9 & {$[43]$} \\
$\sigma_{\mathrm{W}}$ & 0.1 & {$[43]$} \\
$\ell_{\mathrm{p}}{ }^{\mathrm{G}}\left[\mu \mathrm{m}^{2} \mathrm{sec}^{-1} \mathrm{mmHg}^{-1}\right]$ & 0.601854 & {$[43]$} \\
$\ell_{\mathrm{p}}{ }^{\mathrm{W}}\left[\mu \mathrm{m}^{2} \mathrm{sec}^{-1} \mathrm{mmHg}^{-1}\right]$ & 4.15203 & {$[43]$} \\
$\mu[\mathrm{mmHg} \mathrm{sec}]$ & $5.2504 \cdot 10^{-6}$ & {$[49]$} \\
$\mathrm{r}_{\mathrm{s}}\left[\mu \mathrm{m}^{-3}\right]$ & $3.6 \cdot 10^{-3}$ & {$[40]$} \\
$\mathrm{D}_{\text {free }}\left[\mu \mathrm{m}^{2} \mathrm{sec}^{-1}\right]$ & 60 & {$[50]$} \\
$\alpha$ & 1.001 &
\end{tabular}

Table 1. Typical values of the material properties of an intact capillary: $\sigma$ is the reflection coefficient and $\ell_{\mathrm{p}}$ is the hydraulic conductivity. The typical values of capillary blood hydrostatic and osmotic pressures $\left(\mathrm{p}_{\mathrm{c}}\right.$ and $\Pi_{\mathrm{c}}$ ) are reported together with typical values of interstitial pressures $p_{o}$ and $\Pi_{0}$. The superscripts $G$ and $W$ indicate the glycocalyx and the endothelial layers, respectively. $\mu$ is kinematic viscosity of the fluid, $r_{s}$ is the mean molecular radius of human serum albumin and $\mathrm{D}_{\text {free }}$ is its free diffusion coefficient. The coefficient $\alpha>1$ depends on the Péclet number and is defined such as to respect condition (10).

\section{SIMULATION OF GLYCOCALYX SHEDDING}

Degradation and/or loss of glycocalyx have been shown during exposure to atherogenic/cardiovascular risk factors, including inflammatory and atherogenic stimuli, ischemia/reperfusion and oxidized low-density lipoprotein infusion [5]; patients with type 1 diabetes mellitus and microalbuminuria are more susceptible to glycocalyx degradation [16]. Apart from pathological conditions, glycocalyx thickness can change also because of increased shear stress (even at atherosclerosis-prone sites) and in response to agonists such as adenosine, which may increase wall conductivity up to $40 \%$ [51].

In this section, we simulate glycocalyx deterioration, possibly due to physiological glycocalyx fiber rearrangement and/or to enzymatic digestion.

\subsection{Simulating glycocalyx modulation?}

In [16], glycocalyx thickness in sub-lingual capillaries was estimated from the difference between capillary red blood cell column width during baseline (functionally perfused capillary diameter) and after passage of a leukocyte (anatomic capillary diameter). A reduction of about $45 \%$ was found in the glycocalyx thickness of healthy subjects, compared to patients with type 1 diabetes mellitus. This shows indirectly that patients affected by this disease suffer from a reduction of the glycocalyx thickness, since the leukocyte interacts less with the glycocalyx. In addition, an electron microscopic study of mouse carotid arteries [22] showed a thinning of endothelial glycocalyx layer of about $75 \%$ at an atherosclerosis-prone site compared to the glycocalyx thickness at a low-atherogenicrisk site [5].

Moreover, Adamson [11] observed that the treatment of frog mesenteric capillaries with the glycocalyx-degrading enzyme pronase produced a 2.5 -fold increase in the postpronase hydraulic conductivity compared to the initial control value, accompanied by no significant changes in the morphology of the inter-cellular clefts and large alterations in the luminal glycocalyx structure, due to its partial digestion (see also Figure 1 in [8]). This suggests a correlation between the changes in physiologically measured hydraulic conductivity and morphologically demonstrated alterations in the glycocalyx structure. Incidentally, the 2.5-fold increase in capillary permeability (which reaches nearly a 20-fold increase in particular vessels) implies that at least $60 \%$ of the hydraulic resistance to plasma flow across microvessel walls is associated with the endothelial glycocalyx.

According to Equation (7), an increment in glycocalyx hydraulic conductivity may be the consequence of a raise in the number $\mathrm{N}$ of pores, or an increase in their radius $r_{p}$ or of both of them (see Figure 3 in [11]). Here we choose to simulate damage of the glycocalyx by increasing both $\mathrm{N}$ and $r_{p}$. A change in $N$ determines a variation of both $\ell_{p}$ and $\ell_{d}$, but does not alter $\sigma$ since the latter is only related to the ratio of solute and pore size (see Figure 2a). On the other hand, a change in $r_{p}$ produces a change in $\sigma, \ell_{p}$ and $\ell_{d}$.

Figure 2 shows the variation of the physiological parameters $\sigma, \ell_{\mathrm{p}}$ and $\ell_{\mathrm{d}}$ due to changes of both $\mathrm{N}$ and $\mathrm{r}_{\mathrm{p}}$. Notice that all the other quantities are fixed according to the values reported in Table 1 and referring to an intact (not damaged) glycocalyx.

We thus model the deterioration of the endothelial glycocalyx by three means: (i) by decreasing its thickness $\Delta_{\mathrm{G}}$, (ii) by increasing the number $\mathrm{N}$ of its pores and (iii) by augmenting their radii $r_{p}$.

\subsection{Computed fluxes in the case of glycocalyx damage}

Figures 3a-3f show the volume (Figures $3 a, 3 c$ and $3 e$ ) and solute fluxes (Figures 3b, 3d and 3f) made dimensionless with respect to the reference fluxes of an intact vessel wall reported in Table 1 as a function the parameters describing the change of status of the glycocalyx (i.e. $\Delta_{\mathrm{G}}, \mathrm{N}$ and $\mathrm{r}_{\mathrm{p}}$ ). In the figures, these parameters are let to change in pairs, with the remaining parameter kept constant.

Glycocalyx thinning, accompanied by an increase of pore number $\mathrm{N}$ and/or pore radius $\mathrm{r}_{\mathrm{p}}$, result in an increase of plasma filtration and/or solute extravasation, as expected (see the dark grey zones representing the highest values of volume/solute fluxes). 


\section{Reflection coefficient $\sigma$}

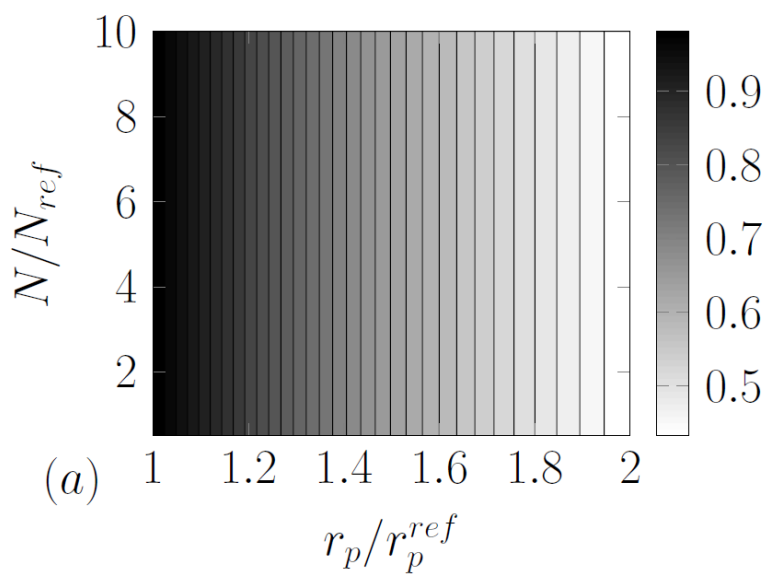

Hydraulic conductivity $\ell_{p}$

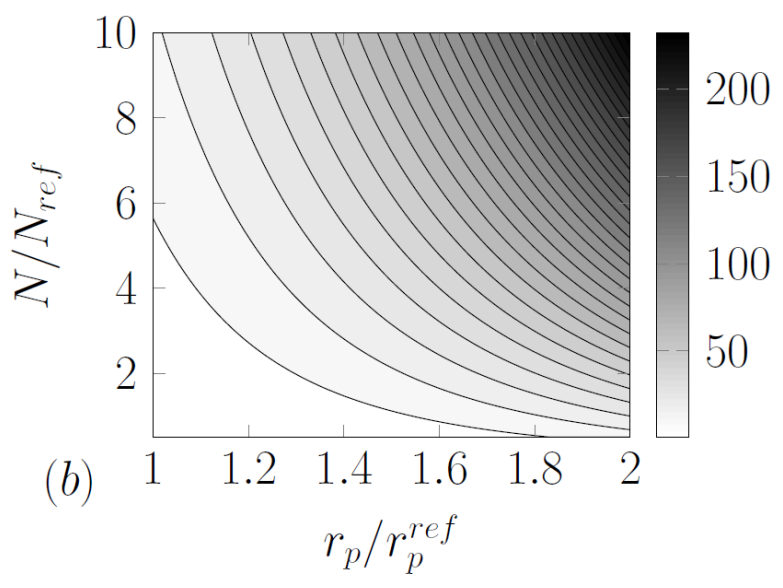

\section{Diffusional permeability $\ell_{d}$}

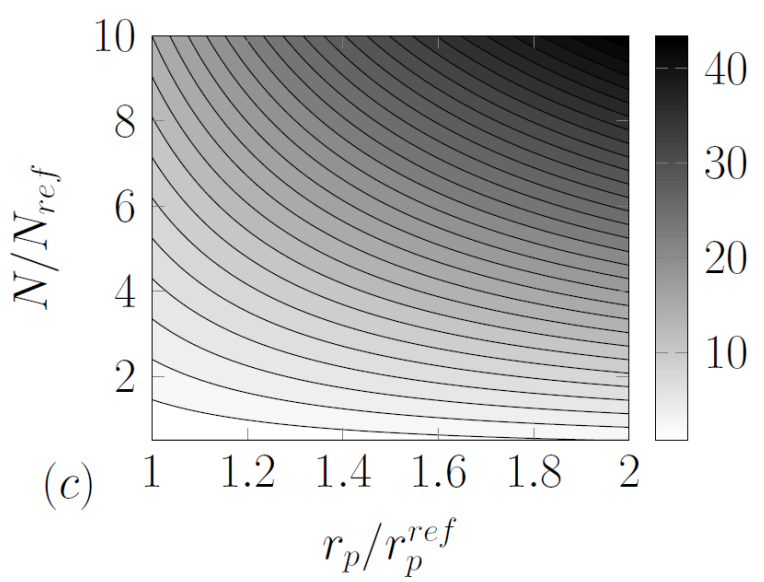

Figure 2. Physiological parameters $\sigma, \ell_{\mathrm{p}}$ and $\ell_{\mathrm{d}}$ (divided by the reference value obtained for an intact vessel wall) varying with respect to the radius $r_{p}$ and number $\mathrm{N}$ of pores of the glycocalyx layer (divided by the corresponding values of an integer glycocalyx).
Notice the 6 to 7 -fold increase of the volume flux in the case in which the mean pore radius $r_{p}$ increases to twice the reference value (see Figures $3 \mathrm{a}$ and $3 \mathrm{e}$ ), whereas it increases up to 3.5 times the reference value when $r_{p}$ is kept fixed and the other two parameters varied within their range of variability (Figure 3c). The influence of $r_{p}$ on the physiological parameters vary among them; see in particular Figure $2 \mathrm{a}$ in which doubling $\mathrm{r}_{\mathrm{p}}$ halves the value of $\sigma$. This suggests a pivotal role of $\sigma$ in the volume flux, being more sensitive to variations of $\sigma$ than of other parameters.

The behavior of the solute flux is similar, except that a change in glycocalyx thickness $\Delta_{\mathrm{G}}$ and/or pore number $\mathrm{N}$ quadruples the flux (see Figure 3d), whereas a change in $r_{p}$ (and thus in $\sigma$ ) decouples this effect, as clearly depicted in Figures $3 \mathrm{~b}$ and $3 \mathrm{f}$, respectively. These results are in line with experimental observations, where a significant increase in macro-molecular flux across microvessel wall was observed both after exposure to $\mathrm{H}_{2} \mathrm{O}_{2}$ [25] and after treatment with heparan-sulfate-degrading enzymes [24]. Depending on the enzymes used, it was noticed a 1.4 to 3 -fold increase in the trans-vascular albumin flux after enzyme-treatment compared to the initial macro-molecular flux, supporting our predictions [24].

The almost horizontal contour lines shown in Figures $3 \mathrm{a}$ and $3 \mathrm{~b}$ are indicative of a small influence of $\Delta_{\mathrm{G}}$ variations on both volume and solute fluxes, if compared with the change caused by variation of $r_{p}$. On the other hand, when $\Delta_{G}$ is kept fixed, volume (Figure 3e) and solute (Figure 3f) fluxes are chiefly controlled by $\mathrm{N}$ and are insensitive to $r_{p}$ variations. The effect of these variations is much larger on the solute flux than on the volumetric flux with about 40-fold increase of the former (see Figures $3 \mathrm{~b}$ and $3 \mathrm{f}$ ) against the maximum of 7-fold increase in the latter. The only case in which variation of volumetric and solute fluxes are comparable is that in which with $r_{p}$ maintained constant $\mathrm{N}$ and $\Delta_{\mathrm{G}}$ are varied spanning their range of variability (Figures $3 c$ and $3 d$ ). In this case, both fluxes are sensitive to both variations, as shown by the inclined contour lines.

\section{INFLUENCE OF PRESSURE INCREASE}

In this section, we study the impact of increasing hydrostatic blood pressure and solute volume fluxes, as in preand post-stenotic blood vessels.

Figures 4a-4f show volume and solute fluxes (normalized with respect to the reference fluxes of an intact vessel, whose properties are listed in Table 1) when one of the parameters describing glycocalyx damage (i.e., $\Delta_{\mathrm{G}} / \Delta_{\mathrm{G}}{ }^{\text {ref }}, \mathrm{N} / \mathrm{N}_{\mathrm{ref}}$ and $\mathrm{r}_{\mathrm{p}} / \mathrm{r}_{\mathrm{p}}{ }^{\text {ref }}$ ), normalized by the reference values show in Table 1 , is varied in combination with the hydrostatic blood pressure $\mathrm{p}_{\mathrm{c}}$, with the other two parameters kept constant. Local hypertension, here represented by $p_{c}$ larger than the reference value for a healthy control, results in an increase of plasma filtration and solute leakage, worsened by glycocalyx shedding (see the dark grey zones representing the highest values of volume/solute fluxes). By halving glycocalyx thickness $\Delta_{\mathrm{G}}$, both fluxes become 5 times larger than the references when blood pressure $\mathrm{p}_{\mathrm{c}}$ is doubles (Figures $4 \mathrm{a}$ and 4b). As expected, both fluxes rise more rapidly with $\mathrm{p}_{\mathrm{c}}$, reaching much higher values, for a thinner glycocalyx. 
Volume flux

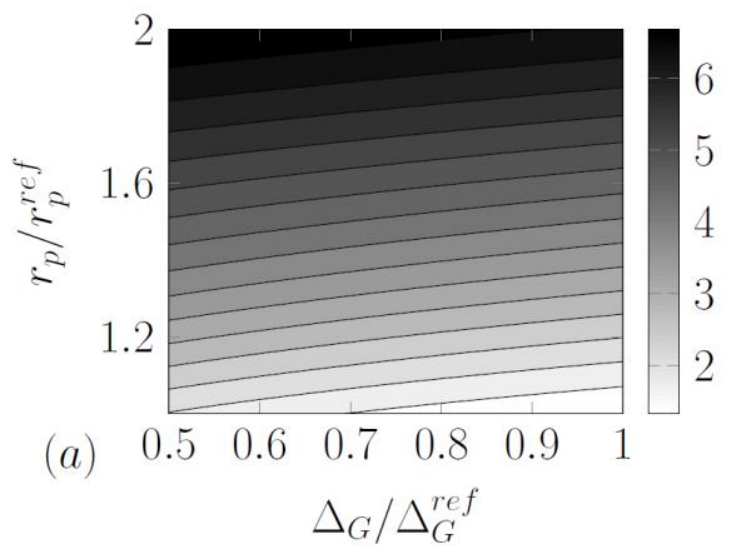

Volume flux



Volume flux

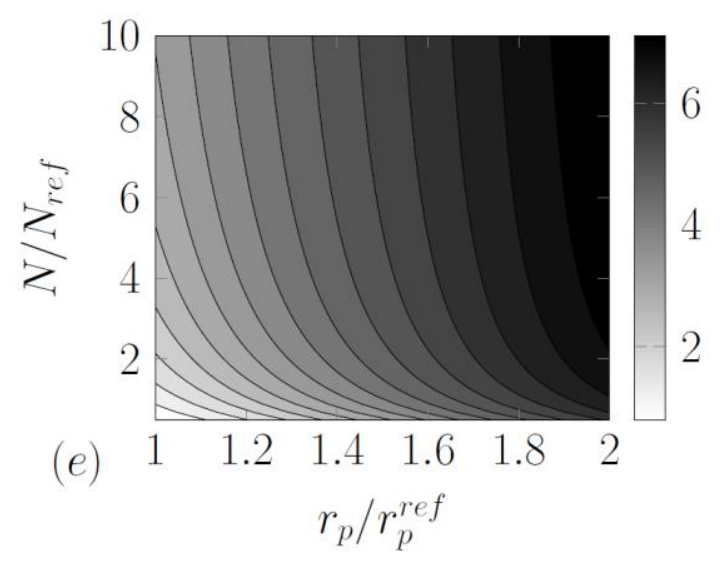

Solute flux

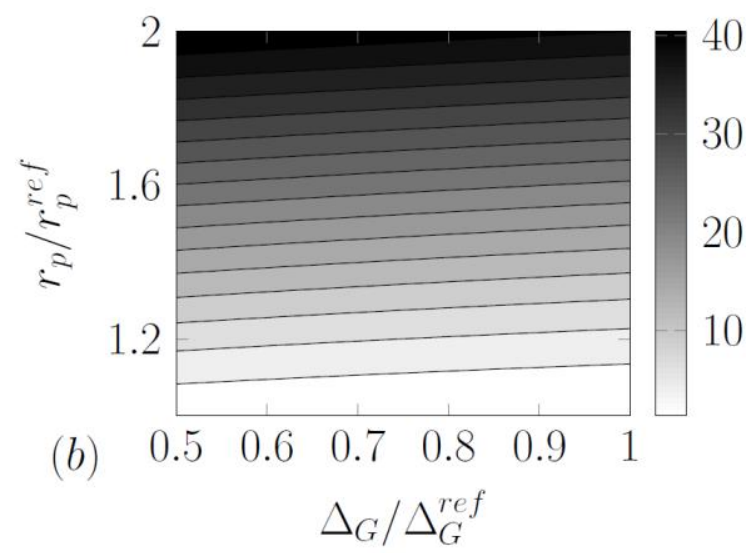

Solute flux

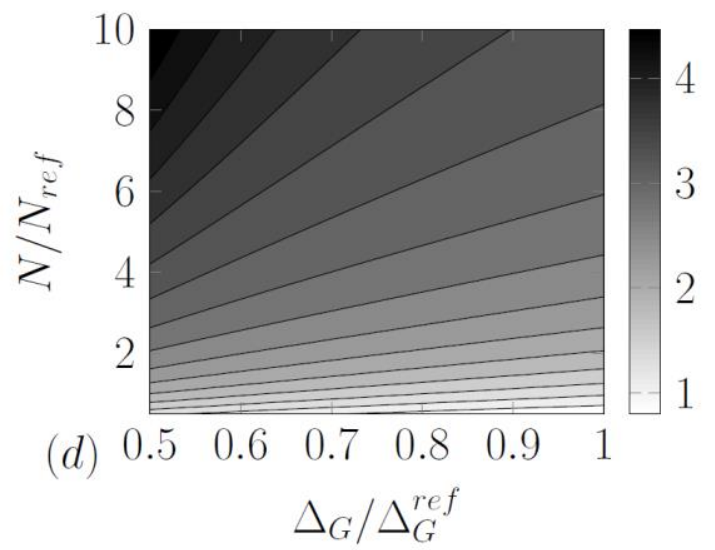

Solute flux

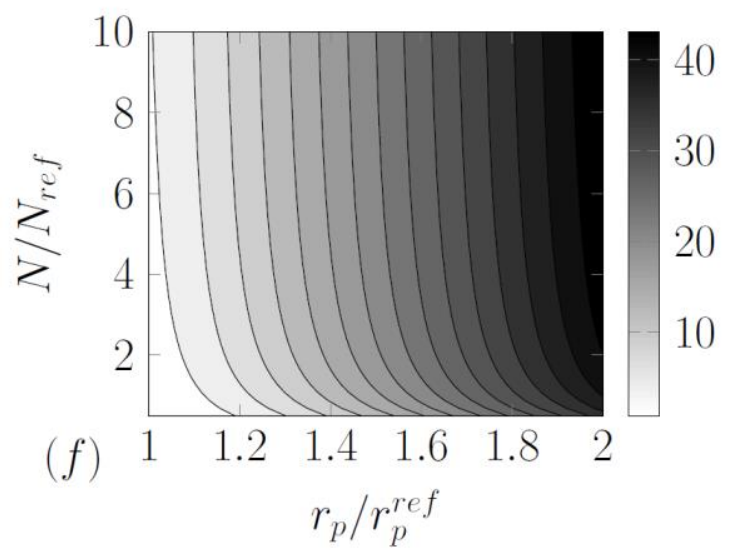

Figure 3. Contour plots of volume and solute fluxes (divided by reference fluxes) as functions of glycocalyx thickness $\Delta_{\mathrm{G}}$, pore radius $r_{p}$ and number of pores $N$ (divided by the corresponding values of an intact glycocalyx). Note the change of scale from panel to panel. 
Volume flux



Volume flux

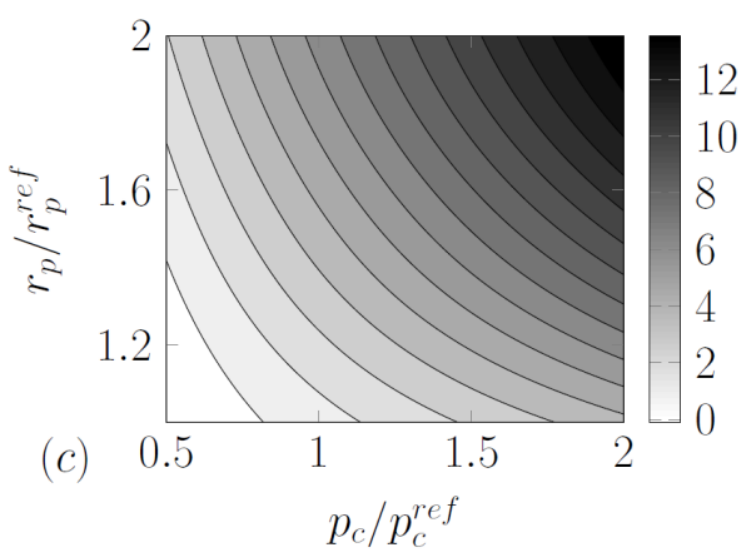

Volume flux

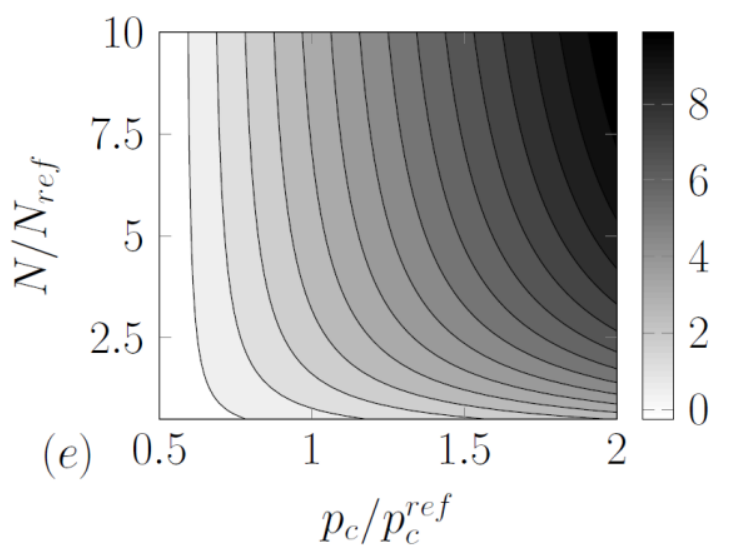

Solute flux

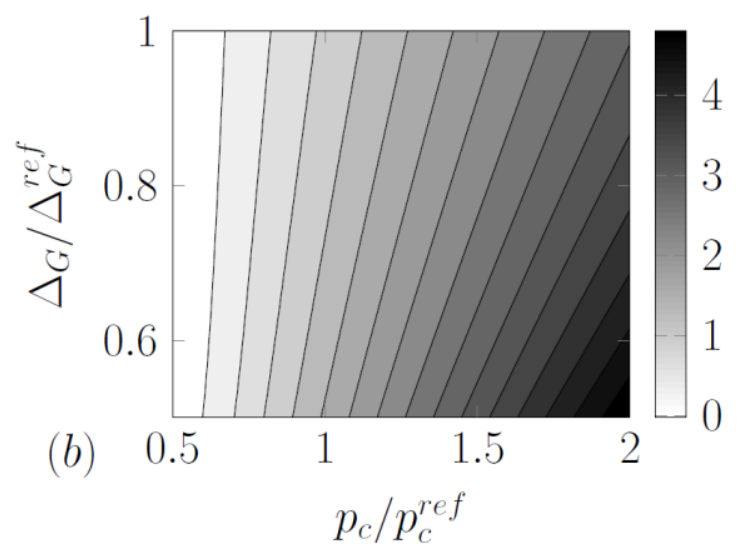

Solute flux



Solute flux

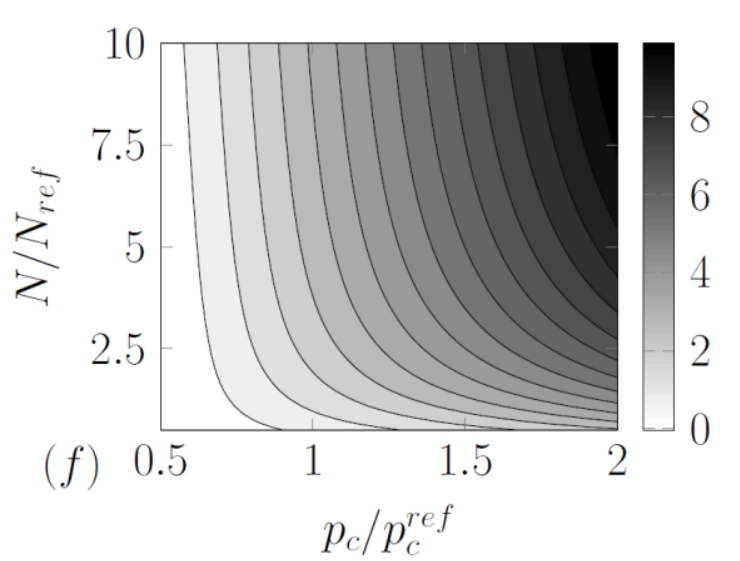

Figure 4. Contour plots of volume and solute fluxes (divided by reference fluxes) as functions of hydrostatic blood pressure $\mathrm{p}_{\mathrm{c}}$ (normalized by its typical value in a microvessel of a healthy control) and the morphological and/or physical parameters $\Delta_{\mathrm{G}}, \mathrm{r}_{\mathrm{p}}$ and $\mathrm{N}$ (normalized by the corresponding values of an intact glycocalyx). Note the change of scale from panel to panel. 
Increasing $r_{p}$, while keeping constant the other two parameters $\left(\Delta_{G}\right.$ and $\left.N\right)$ causes a large increase of both fluxes, which reach values much higher than similar changes of $r_{p}$ with $p_{c}$ as can be noticed by comparing Figure $4 c$ with Figures $3 \mathrm{a}$ and $3 \mathrm{e}$ for the volume flux and Figure $4 \mathrm{~d}$ with Figures $3 \mathrm{~b}$ and $3 \mathrm{f}$, for the solute flux. A similar variation of $\mathrm{p}_{\mathrm{c}}$, but with changing $\mathrm{N}$ instead of $r_{p}$ produces a smaller increase in the fluxes (Figures $4 \mathrm{e}$ and $4 \mathrm{f}$ ). Notice that while the volumetric flux is in this case still large with respect to the values observed when $\mathrm{N}$ is increased by keeping $\mathrm{p}_{\mathrm{c}}$ constant, as shown in Figures $3 \mathrm{c}$ and $3 \mathrm{e}$, the solute flux reduces significantly with respect to the similar cases shown in Figures $3 \mathrm{~d}$ and $3 \mathrm{f}$. In general, volume and solute fluxes are more sensitive to changes of $p_{c}$ at large values of $N$ and $r_{p}$. The disproportional increase of the solute flux with respect to the parallel increase of the volumetric flux observed when both $\mathrm{p}_{\mathrm{c}}$ and $r_{p}$ are increased, as shown in Figures $4 c$ and $4 d$, in in line with the experimental findings of Valenzuela-Rendon and Manning [52], showing that trans-capillary flux of fluid and proteins in conscious dogs increase both during volumeloading hypertension and during angiotensin II-induced hypertension. In fact, in their first experiment of volumeloading-induced hypertension (with an increase in mean arterial pressure of $24 \%$ ), the fluid flux increased of $165 \%$, whereas the protein flux, apparently dominated by convection, increased only by $57 \%$ [52]. Analogously, during angiotensin II-induced hypertension experiment (with an increase in mean arterial pressure of $45 \%$ ), trans-capillary flux increased by $45 \%$ and protein flux increased by $34 \%$ [53]. Our results are in line with these experimental findings, though we cannot apply our model to this case because the Péclet number is not reported $[52,53]$.

Notice that a change in mean arterial pressure is not directly correlated to capillary pressure, since the latter is also affected by nervous control, venous pressure, vascular resistance, gravity and distance along the capillary axis [54]. Nevertheless, in both experiments discussed in [52, 53], hypertension produces an increase in both volume and solute fluxes, as qualitatively reproduced by our model.

\section{DISCUSSION}

Only recently the role of glycocalyx and the adverse effects of its deterioration in several diseases has been accepted [55], for instance, its dysfunction contributes to renal and cardiovascular disease progression, associated with albuminuria [26] and its degradation is recognized in septis with the onset of acute lung injury in most severe cases [56]. In acute lung injury and acute respiratory distress syndrome, pulmonary edema was observed, correlated to glycocalyx deterioration [57]. To the best of our knowledge, myocardial tissue edema, and in general extravascular edema, are measured through the change in pericapillary space [8], without reporting the precise values of plasma and solute fluxes. An increase in pericapillary space by about $65 \%$ is thus measured during myocardial tissue edema, proving fluid and protein extravasation [8]. The results of our model are in line with these observations. With glycocalyx deterioration simulated by reducing its thickness or increasing the radius and/or the number of the pores, the plasma filtration increases from 3 to 7 times, with respect to the reference value (for an intact glycocalyx), while the solute extravasation increases from 4 to 40 times the reference value (see Figures 3a-3f).

A recent study [55] observed a $40 \%$ increase in cardiac microvascular perfusion in control rats after adenosine infusion, whereas neither microvascular dysfunction nor loss of glycocalyx integrity was observed upon adenosine infusion. This suggests an important role of the level of insulin resistance in diabetes, which, by now, is neglected in our mathematical model.

Our model is also able to capture the role of capillary pressure in transvascular homeostasis, especially in the presence of glycocalyx degradation, as shown by the increase of plasma filtration from 5 to 14 times accompanied by an increase of solute extravasation of 5 to 80 times, depending on the type of damage considered (see Figures 4a-4f). This occurs, for example, in patients affected by hypertensive retinopathy, where the retinal vasculature may be sufficiently injured to cause occlusion or leakage, thus causing extravascular edema and intraretinal hemorrhage in the most severe cases. In addition, hypertensive optic neuropathy accompanied by severe hypertension correlates to flame hemorrhage and optic disc edema [28]. Another confirmation of our findings can be seen in the brain: even a slight increase in pressure may produce serious consequences, as observed in the experiments of Mayhan and Heistad [58], where an increase in cerebral venous pressure either due to phenylephrine-induced hypertension or to superior venae cavae occlusion was shown to cause the break of rat cerebral capillaries. Furthermore, our work can possibly explain the recent association of diabetes with proteinuria and a highly increased risk of atherosclerosis development: diabetes is often associated to glycocalyx degradation [16] and it has recently been hypothesized to accelerate transvascular transport of both albumin and lipoproteins by $25 \%$ and $28 \%$, respectively [59]. Moreover, this increased transvascular solute transport is accelerated in the presence of systolic hypertension or albuminuria, where solute flux of albumin and low-density lipoprotein rises by $29 \%$ and $44 \%$, respectively, compared to healthy controls. This agrees with our predictions of a slackening of the vascular solute barrier in the case of glycocalyx degradation, as in the case of diabetic patients, even worsened in the presence of hypertension.

In the present work, we neglected the role of the lymphatic system, which drains accumulated ultrafiltrate, as demonstrated by the increase in its lymph flow by 2-3 times in the thoracic duct during volume-loading hypertension [52]. Indeed, a well-known maneuver against edema is the increase of lymph flow until it exceeds the trans-capillary value, thus limiting fluid accumulation in the surrounding tissue [54]. To model this highly dynamic process, a mathematical description of tissue morphology and transport properties is needed, together with a suitable model of the time-dependent pressure changes in the lymphatic capillaries. This is outside the scope of the present paper.

Moreover, traditionally the brain been thought to lack a lymphatic system, because since recently none observed lymphatic vessels within the brain. However, a recent study demonstrated the presence of a lymphatic system also within the brain [60], thereby calling for accurate studies to 
understand the extent of the effects on physiological and pathological alterations in cerebral haemodynamics.

The trans-vascular model proposed in the present work can be extended in many ways, for instance by coupling it to haemodynamics, since plasma filtration and solute transport are closely linked to local blood flow dynamics. Actually, it has been observed that vessel occlusion, resulting in anomalous blood flow and hypertension, is associated with the tendency to edema formation and perivascular accumulation of material $[58,61]$. The aim of trans-vascular transport studies is to determine the influence of disturbed flow patterns first on luminal pressure and then on the local concentration of substances both in the lumen and in the vessel wall.

A recent study [62] has shown that extra-cranial venous strictures can lead to pressure increases in intra-cranial veins. Our model can be easily formulated to model the blood-brain barrier by adding other three layers, representing the pericytes, the basement membrane and the astrocyte feet [63]. The difficulty here is in obtaining the phenomenological parameters of these new layers, rather than in the geometrical complexity, since a general solution for any number of layers can be obtained [38]. This would result in a better understanding of neuro-degenerative conditions, which seem to be connected to vascular and/or transport anomalies, such as Parkinson's disease, Alzheimer's disease and multiple sclerosis [63, 49]. Drug delivery in the treatment of diseases could also be explored and potentially optimized by extending the current models.

Since all these hypotheses are controversial, accurate mathematical modeling may provide valuable support in elucidating the relevant mechanisms leading to a disease and possibly aiding the process of designing appropriate cures.

\section{CONCLUSION}

We have applied our multi-layer steady-state mathematical model to simulate the impact of glycocalyx degeneration, due to its thinning and/or alteration on volumetric and solute fluxes across microvessel walls.

Our simulations showed that plasma and solute fluxes across microvessel walls are impacted by the mean pore radius representing glycocalyx porosity more than glycocalyx thickness and pore number. This suggests a significant influence of glycocalyx reflection coefficient, which depends on the ratio between the radius of solute's molecule and the pore radius, on transport processes.

Our model is also able to quantify the effects of hypertension on both fluxes, also when glycocalyx is deteriorated; our simulations are in qualitative agreement with the experimental results reported in the literature, where both edema and hemorrhage are observed.

To take into account also the dynamic changes in lymph flow and tissue properties, a time-dependent mathematical model of trans-vascular phenomena is needed. A forthcoming work has the objective to study this.

To summarize, our simple, yet effective, model provides results that are in line with physiological studies and can possibly be a useful tool in the prediction of the long-term effect of glycocalyx deterioration and/or hypertension on a number of diseases.

\section{ACKNOWLEDGEMENTS}

This work has been partially funded by Fondazione Cassa $\mathrm{di}$ Risparmio di Trento e Rovereto (CARITRO, Italy), project no. 2011.0214.

\section{REFERENCES}

[1] Sugihara-Seki M, Fu BM. Blood flow and permeability in microvessels. Fluid Dyn Res 2005; 37:82-132.

[2] Weinbaum S, Tarbell JM, Damiano ER. The structure and function of the endothelial glycocalyx layer. Annu Rev Biomed Eng 2007; 9:121-167.

[3] Squire JM, Chew M, Nneji G, Neal C, Barry J, Michel C. Quasi-periodic substructure in the microvessel endothelial glycocalyx: a possible explanation for molecular filtering? J Struct Biol 2001; 136(3):239-255.

[4] Arkill KP, Neal CR, Mantell JM, et al. 3D Reconstruction of the Glycocalyx Structure in Mammalian Capillaries using Electron Tomography. Microcirculation 2012; 19:343-351.

[5] VanTeeffelen JWGE, Brands J, Stroes ES, Vink H. Endothelial Glycocalyx: Sweet Shield of Blood Vessels. Trends Cardiovasc Med 2007; 17:101-105.

[6] Weinbaum S, Zhang XB, Han YF, Vink H, Cowin SC. Mechanotransduction and flow across the endothelial glycocalyx. Proc Natl Acad Sci USA 2003; 100:7988-7995.

[7] Henry CB, Duling BR. Permeation of the luminal capillary glycocalyx is determined by hyaluronan. Am J Physiol 1999; 277:H508-H514.

[8] van den Berg BM, Vink H, Spaan JAE. The Endothelial Glycocalyx Protects Against Myocardial Edema. Circ Res 2003; 92:592-594.

[9] Florian JA, Kosky JR, Ainslie K, Pang Z, Dull RO, Tarbell JM. Heparan sulfate proteoglycan is a mechanosensor on endothelial cells. Circ Res 2003; 93:e136-e142.

[10] Mochizuki S, Vink H, Hiramatsu O, et al. Role of hyaluronic acid glycosaminoglycans in shear-induced endothelium-derived nitric oxide release. Am J Physiol Heart Circ Physiol 2003; 285:H722-H726.

[11] Adamson RH. Permeability of frog mesenteric capillaries after partial pronase digestion of the endothelial glycocalyx. J Physiol 1990; 428:1-13.

[12] Huxley VH, Williams DA. Role of a glycocalyx on coronary arteriole permeability to proteins: evidence from enzyme treatments. Am J Physiol Heart Circ Physiol 2000; 278:H1177-H1185.

[13] Jeansson M, Haraldsson B. Glomerular size and charge selectivity in the mouse after exposure to glucosaminoglycan-degrading enzymes. J Am Soc Nephrol 2003; 14:1756-1765.

[14] Constantinescu AA, Vink H, Spaan JA. Endothelial cell glycocalyx modulates immobilization of leukocytes at the endothelial surface. Arterioscler Thromb Vasc Biol 2003; 23:1541-1547.

[15] Tarbell JM, Cancel LM. The glycocalyx and its significance in human medicine. J Intern Med 2016; doi: 10.1111/joim.12465. 
[16] Nieuwdorp M, Mooij HL, Kroon J, et al. Endothelial glycocalyx damage coincides with microalbuminuria in type 1 diabetes. Diabetes 2006; 55(4):1127-1132.

[17] Martens RJ, Vink H, van Oostenbrugge RJ, Staals J. Sublingual microvascular glycocalyx dimensions in lacunar stroke patients. Cerebrovasc Dis $2013 ; 35(5): 451-454$.

[18] Nieuwdorp M, Holleman F, de Groot E, et al. Perturbation of hyaluronan metabolism predisposes patients with type 1 diabetes mellitus to atherosclerosis. Diabetologia 2007; 50(6):1288-1293.

[19] Vlahu CA, Lemkes BA, Struijk DG, Koopman MG, Krediet RT, Vink H. Damage of the Endothelial Glycocalyx in Dialysis Patients. J Am Soc Nephrol 2012; 23:1900-1908.

[20] Marechal X, Favory R, Joulin O, et al. Endothelial glycocalyx damage during endotoxemia coincides with microcirculatory dysfunction and vascular oxidative stress. Shock 2008; 29(5):572-576.

[21] Donati A, Damiani E, Domizi R, et al. Alteration of the sublingual microvascular glycocalyx in critically ill patients. Microvasc Res 2013; S0026-2862(13):00149-0.

[22] van den Berg BM, Spaan JAE, Rolf TM, Vink H. Atherogenic region and diet diminish glycocalyx dimension and increase intima-to-media ratios at murine carotid artery bifurcation. Am J Physiol Heart Circ Physiol 2006; 290:H915-H920.

[23] Constantinescu AA, Spaan JAE, Arkenbout EK, Vink H, VanTeeffelen JWGE. Degradation of the endothelial glycocalyx is associated with chylomicron leakage in mouse cremaster muscle microcirculation. Thromb Haemost 2011; 105:790-801.

[24] Singh A, Satchell S, Neal CR, McKenzie EA, Tooke JE, Mathieson PW. Glomerular endothelial glycocalyx constitutes a barrier to protein permeability. J Am Soc Nephrol 2007; 18(11):2885-2893.

[25] Singh A, Ramnath RD, Foster RR, et al. Reactive oxygen species modulate the barrier function of the human glomerular endothelial glycocalyx. PLoS One 2013; 8(2):e55852.

[26] Rabelink TJ, de Zeeuw D. The glycocalyx-linking albuminuria with renal and cardiovascular disease. Nat Rev Nephrol 2015; 11(11):667-676.

[27] Mulivor AW, Lipowsky HH. Inflammation- and ischemia-induced shedding of venular glycocalyx. Am J Physiol Heart and Circulatory Physiology 2004; 286(5):H1672-H1680.

[28] Chobanian AV, Bakris GL, Black HR, et al. Seventh report of the Joint National Committee on Prevention, Detection, Evaluation, and Treatment of High Blood Pressure. Hypertension 2003; 42(6):1206-1252.

[29] Curry FE, Michel CC. A fiber matrix model of capillary permeability. Microvasc Res 1980; 20(1):96-99.

[30] Tsay R, Weinbaum S. Viscous flow in a channel with periodic crossbridging fibres: exact solutions and Brinkman approximation. J Fluid Mech 1991; 226:125-148.

[31] Weinbaum S, Tsay R, Curry FE. A three-dimensional junction-porematrix model for capillary permeability. Microvasc Res 1992; 44(1):85-111.

[32] Bundgaard M. The three-dimensional organization of tight junctions in a capillary endothelium revealed by serial-section electron microscopy. J Ultrastruct Res 1984; 88(1):1-17.
[33] Ward BJ, Bauman KF, Firth JA. Interendothelial junctions of cardiac capillaries in rats: their structure and permeability properties. Cell Tissue Res 1988; 252(1):57-66.

[34] Fu BM, Weinbaum S, Tsay RY, Curry FE. A junction-orifice-fiber entrance layer model for capillary permeability: application to frog mesenteric capillaries. J Biomech Eng 1994; 116:502-513.

[35] Michel CC, Curry FE. Microvascular Permeability. Physiol Rev 1999; 79(3):703-761

[36] Fu BM, Chen B, Chen W. An electrodiffusion model for effects of surface glycocalyx layer on microvessel permeability. Am J Physiol Heart Circ Physiol 2003; 284(4):H1240-H1250.

[37] Speziale S, Sivaloganathan S. Poroelastic theory of transcapillary flow: Effects of endothelial glycocalyx deterioration. Microvasc Res 2009; 78:432441.

[38] Facchini L, Bellin A, Toro EF. A mathematical model for filtration and macromolecule transport across capillary walls. Microvasc Res 2014; 94(C):52-63.

[39] Freeze RA. A stochastic-conceptual analysis of one-dimensional groundwater flow in nonuniform homogeneous media. Water Resour Res 1975 11(5):725-741.

[40] Levick JR, Michel CC. Microvascular fluid exchange and the revised Starling principle. Cardiovasc Res 2010; 87(2):198-210.

[41] Renkin EM. Filtration, diffusion, and molecular sieving through porous cellulose membranes. J Gen Physiol 1954; 38(2):225-243.

[42] Katchalsky A, Curran PF. Non-equilibrium Thermodynamics in Biophysics. Harvard Univ. Press 1965

[43] Speziale S, Tenti G, Sivaloganathan S. A poroelastic model of transcapillary flow in normal tissue. Microvasc Res 2008; 75(2):285-295.

[44] Vink H, Duling BR. Capillary endothelial surface layer selectively reduces plasma solute distribution volume. Am J Physiol Heart Circ Physiol 2000; 278(1):H285-H289.

[45] Taylor G. Dispersion of Soluble Matter in Solvent Flowing Slowly through a Tube. Proc R Soc Lond A Math Phys Sci 1953; 219(1137):186203.

[46] Charm SE, Kurland GS. Blood Flow and Microcirculation. New York: Wiley 1974.

[47] Adamson RH, Lenz JF, Zhang X, Adamson GN, Weinbaum S, Curry FE. Oncotic pressures opposing filtration across non-fenestrated rat microvessels. J Physiol 2004; 557(3):889-907.

[48] Levick JR. Capillary filtration-absorption balance reconsidered in light of dynamic extravascular factors. Exp Physiol 1991; 76:825-857.

[49] Li G, Yuan W, Fu BM. A model for the blood-brain barrier permeability to water and small solutes. J Biomech 2010; 43(11):2133-2140.

[50] Stevens AP, Hlady V, Dull RO. Fluorescence correlation spectroscopy can probe albumin dynamics inside lung endothelial glycocalyx. Am J Physiol Lung Cell Mol Physiol 2007; 293(2):L328-L335.

[51] VanTeeffelen JW, Dekker S, Fokkema DS, Siebes M, Vink H, Spaan JA Hyaluronidase treatment of coronary glycocalyx increases reactive hyperemia but not adenosine hyperemia in dog hearts. Am J Physiol Heart Circ Physiol 2005; 289:H2508-H2513. 
[52] Valenzuela-Rendon J, Manning RDJ. Chronic transvascular fluid flux and lymph flow during volume-loading hypertension. Am J Physiol 1990; 258:H1524-H1533.

[53] Valenzuela-Rendon J, Manning RDJ. Chronic lymph flow and transcapillary fluid flux during angiotensin II hypertension. Am J Physiol 1990; 259:R1205-R1213.

[54] Levick JR. An Introduction to Cardiovascular Physiology. CRC Press 2010.

[55] van Haare J, Kooi ME, Vink H, et al. Early impairment of coronary microvascular perfusion capacity in rats on a high fat diet. Cardiovasc Diabetol 2015; 14:150.

[56] Schmidt EP, Yang Y, Janssen WJ, et al. The pulmonary endothelial glycocalyx regulates neutrophil adhesion and lung injury during experimental sepsis. Nat Med 2012; 18(8):1217-1223.

[57] Yang Y, Schmidt EP. The endothelial glycocalyx: An important regulator of the pulmonary vascular barrier. Tissue Barriers 2013; 1(1):e23494.
[58] Mayhan WG, Heistad DD. Role of veins and cerebral venous pressure in disruption of the blood-brain barrier. Circ Res 1986; 59:216-220.

[59] Jensen JS, Feldt-Rasmussen B, Borch-Johnsen K, Jensen KS, Nordestgaard BG. Increased transvascular lipoprotein transport in diabetes: association with albuminuria and systolic hypertension. J Clin Endocrinol Metab 2005; 90(8):4441-4445.

[60] Louveau A, Smirnov I, Keyes TJ, et al. Structural and functional features of central nervous system lymphatic vessels. Nature 2015; 523(7560):337341 .

[61] Singh A, Zamboni P. Anomalous venous blood flow and iron deposition in multiple sclerosis. J Cereb Blood Flow Metab 2009; 29(12):1867-1878.

[62] Müller LO, Toro EF, Haacke EM, Utriainen D. Impact of CCSVI on cerebral haemodynamics: a mathematical study using MRI angiographic and flow data. Phlebology 2015; doi: 10.1177/0268355515586526.

[63] Hawkins B, Davis T. The blood-brain barrier/neurovascular unit in health and disease. Pharmacol Rev 2005; 57(2):173-185. 